\title{
Cluster observations of trapped ions interacting with magnetosheath mirror modes
}

\author{
J. Soucek ${ }^{1,2}$ and C. P. Escoubet ${ }^{1}$ \\ ${ }^{1}$ ESTEC, European Space Agency, Keplerlaan 1, 2201 AZ, Noordwijk, The Netherlands \\ ${ }^{2}$ Institute of Atmospheric Physics, Bocni II 1401, 14131 Prague, Czech Republic
}

Received: 4 March 2011 - Revised: 17 May 2011 - Accepted: 26 May 2011 - Published: 16 June 2011

\begin{abstract}
Mirror modes are among the most intense low frequency plasma wave phenomena observed in the magnetosheaths of magnetized planets. They appear as large amplitude non-propagating fluctuations in the magnetic field magnitude and plasma density. These structures are widely accepted to represent a non-linear stage of the mirror instability, dominant in plasmas with large ion beta and a significant ion temperature anisotropy $T_{\perp} / T_{\|}>1$. It has long been recognized that the mirror instability both in the linear and non-linear stage is a kinetic process and that the behavior of resonant particles at small parallel velocities is crucial for its development and saturation. While the dynamics of the instability and the effect of trapped particles have been studied extensively in theoretical models and numerical simulations, only spurious observations of the trapped ions were published to date. In this work we used data from the Cluster spacecraft to perform the first detailed experimental study of ion velocity distribution associated with mirror mode oscillations. We show a conclusive evidence for the predicted cooling of resonant ions at small parallel velocities and heating of trapped ions at intermediate pitch angles.
\end{abstract}

Keywords. Magnetospheric physics (Magnetosheath) Space plasma physics (Wave-particle interactions)

\section{Introduction}

Mirror modes are a well known phenomenon occurring in a variety of space plasmas. Observations of these large amplitude plasma structures were reported since the early spacecraft missions from the magnetosheath of the Earth (Kaufmann et al., 1970). They were later discovered in magnetospheres of other planets (Tsurutani et al., 1982), in the

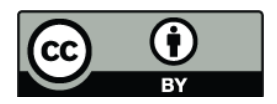

Correspondence to: J. Soucek

(soucek@ufa.cas.cz) solar wind (Tsurutani et al., 1992), in cometary tails (Russell et al., 1987), and more recently also in the heliosheath beyond the termination shock (Burlaga et al., 2006).

They are typically observed as large amplitude nonpropagating variations in magnetic field magnitude accompanied by an anti-correlated variation plasma pressure. The shape of the structures ranges from quasi-sinusoidal oscillations to coherent structures in the form of magnetic peaks (also called humps) or dips (holes, troughs). For more details see Soucek et al. (2008); Génot et al. (2009) and references therein. These structures are generally believed to be generated by mirror instability (Vedenov and Sagdeev, 1958; Hasegawa, 1969) in plasmas with sufficient ion temperature anisotropy. While mirror structures (in particular the magnetic holes) can exist under mirror-stable conditions (Soucek et al., 2008; Génot et al., 2009), unstable conditions must be present at the point of their origin. In the dayside magnetosheath, if plasma $\beta$ is sufficiently high, mirror structures are generated behind the bow shock, where the heated plasma often satisfies the mirror instability threshold condition $\beta_{\perp}\left(T_{\perp} / T_{\|}-1\right)>1$ (Hasegawa, 1969; Hellinger, 2007). The mirror structures are very important for the global magnetosheath dynamics under high $\beta$ conditions: they are responsible for dissipation of the temperature anisotropy excess generated at the bow shock, keeping the magnetosheath plasma in a marginally stable state (Fuselier et al., 1994; Hellinger et al., 2003).

Since the first theoretical works on mirror instability (Vedenov and Sagdeev, 1958; Tajiri, 1967), the importance of kinetic effects in the physics of the process has been recognized. Specifically, the dynamics of particles with small parallel velocities $v_{\|}$resonant with the wave is of critical importance for the initial linear and quasi-linear development of the instability as has been demonstrated by theoretical models (Southwood and Kivelson, 1993; Pantellini and Schwartz, 1995; Hellinger et al., 2009; Pokhotelov et al., 2008, 2010) and numeric simulations (McKean et al., 1993;

Published by Copernicus Publications on behalf of the European Geosciences Union. 

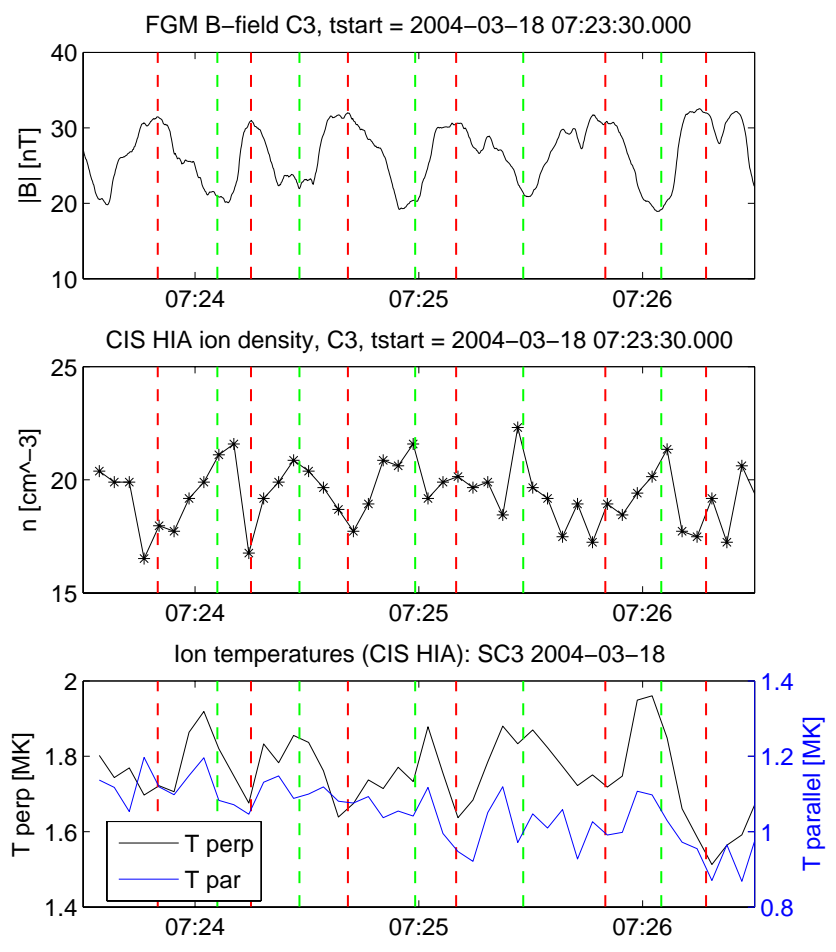

Fig. 1. Magnetic field and ion data for the studied interval from Cluster 3. Top: magnetic field (FGM), Center: ion density (CIS - HIA), Bottom: Perpendicular and Parallel ion temperatures (CIS - HIA). The vertical dashed lines mark the positions of peaks and troughs for easier orientation.

Califano et al., 2008). The more complicated problem of fully non-linear mirror structures has been approached by phenomenological models of particle behavior (Kivelson and Southwood, 1996) and numeric simulations (Califano et al., 2008) which demonstrated the importance of trapped particles for instability saturation at large amplitudes. The above studies predict that the trapped resonant particle population at small $v_{\|}$(pitch angle $\alpha$ close to $90^{\circ}$ ) is being significantly cooled in the early stage of non-linear development of the instability, reducing its growth rate. On the other hand, nonresonant ions at intermediate pitch angles are heated as the instability develops.

A number of experimental works addressed the mirror modes using in-situ spacecraft data (for reviews see Schwartz et al., 1996, and Tsurutani et al., 2011). A large majority of those studies, however, used either no ion measurements or only the measured moments of the distribution function. While these measurements provide valuable information on the bulk plasma status, and were sufficient for many tasks, they do not capture the dynamics of the trapped particles discussed above. To our knowledge, only two studies (Leckband et al., 1995; Fazakerley et al., 1995) were published so far, attempting to resolve these resonant ions in experimental data with partial success. Their results are discussed in detail in Sect. 4. However, similar pitch angle dependent modifications of the trapped population were observed in the electron distribution observed by the AMPTE UKS spacecraft (Chisham et al., 1998).

In this work we use ion and magnetic data from the Cluster spacecraft (Escoubet et al., 2001) to investigate the details of the ion distribution observed within large amplitude mirror structures. We identify the signatures of both the heating and cooling of trapped particles inside the troughs as well as enhancements in particle population at perpendicular pitch angles around the magnetic peaks.

\section{Dataset and data analysis}

The features of the distribution associated with mirror structures are demonstrated on a single interval of data measured by Cluster on 18 March 2004 between 07:23:30 and 07:26:30 UTC. During this interval, Cluster was in the dayside magnetosheath and observed large amplitude mirror structures in the form of a series of magnetic peaks and troughs. This interval of mirror mode activity was chosen because Cluster was in burst mode, transmitting full 3-D ion distributions measured by the CIS instrument (Rème et al., 1997) at 4-s resolution. Ion data from Cluster 3 are shown in this article, but the other spacecraft from which CIS-HIA data were available (Cluster 1) observed very similar features.

Magnetic field from the FGM instrument (Balogh et al., 2001) together with ion density and temperatures from the HIA sensor of the CIS instrument for the chosen interval are plotted in Fig. 1. In this interval Cluster observed nonsinusoidal field variations with $|B|$ ranging between $18 \mathrm{nT}$ and $32 \mathrm{nT}$ and corresponding anti-correlated variations in ion density characteristic for pressure balanced mirror structures. The field oscillations demonstrate the so-called bistability, where the field magnitude alternates between two quasi-stable "states", one associated with low field magnitude around $20 \mathrm{nT}$ and high ion density up to $22 \mathrm{~cm}^{-3}$, the other with a high field level $(\sim 30 \mathrm{nT})$ and lower density $\left(\sim 17-19 \mathrm{~cm}^{-3}\right)$ (Erdôs and Balogh, 1996). The average ion flow velocity of magnetosheath plasma during this interval, measured by the HIA sensor of the CIS instrument on Cluster 3, was $v_{\mathrm{f}}=120 \pm 20 \mathrm{~km} \mathrm{~s}^{-1}$; plasma ion $\beta_{\perp}$ parameter varied between 0.9 and 3.5 with a mean value of $\mu\left(\beta_{\perp}\right)=1.8$.

The bottom panel of Fig. 1 shows an anti-correlation between magnetic field magnitude and perpendicular ion temperature and to lesser extent also parallel ion temperature. The respective correlation coefficients for this dataset are: $\rho(|B|, n)=-0.7, \rho\left(|B|, T_{\perp}\right)=-0.68, \rho\left(|B|, T_{\|}\right)=-0.36$. This increase in overall ion temperature (calculated as a second order moment of the ion distribution) in areas of weaker magnetic field has been described in previous studies (Winterhalter et al., 1995; Balikhin et al., 2010). Later in this article we discuss the origin of this temperature variation 
and show that particles trapped in magnetic troughs are at least partly responsible for this effect.

Simple multi-spacecraft analysis was performed in order to estimate the orientation, velocity and spatial scale of the structures, using the technique described in Horbury et al. (2004). The time lags between spacecraft were calculated by maximizing the inter-spacecraft cross-correlation of magnetic field magnitude (at $0.2 \mathrm{~s}$ resolution). Assuming planarity of the structure, we calculated a unit vector $\boldsymbol{n}$ normal to the planar structure (Eq. 1 of Horbury et al., 2004), and the velocity of the structure in the direction of $\boldsymbol{n}$ in the spacecraft reference frame $v_{\mathrm{n}}$. The analysis yielded a normal velocity of $v_{\mathrm{n}}=53.8 \mathrm{~km} \mathrm{~s}^{-1}$ inclined to the plasma flow velocity at an angle of $60^{\circ}$. Comparing this number to the projection of the plasma flow velocity to the normal direction $\left(\boldsymbol{v}_{\mathrm{f}} \cdot \boldsymbol{n}=56.7 \mathrm{~km} \mathrm{~s}^{-1}\right)$ we can verify that the structure is not propagating with respect to the plasma (within experimental margins) in agreement with the conclusions of Horbury et al. (2004).

The knowledge of the normal direction and velocity allows to calculate reliably the width of the structures in the normal direction by converting the temporal duration of the observed signature to a spatial width. For simplicity, we defined the width of a peak or trough as the distance between points where the magnetic field magnitude crossed the mean value of $\mu(|\boldsymbol{B}|)=25.4 \mathrm{nT}$. After multiplication by $v_{\mathrm{n}}$, we obtain structure widths between $470 \mathrm{~km}$ and $1350 \mathrm{~km}$ or when expressed in local ion Larmor radii between $7 r_{\mathrm{L}}$ and $30 r_{\mathrm{L}}$ (here we used a local value of Larmor radius for ions with thermal energy, calculated at a 4-s time resolution from perpendicular temperature and magnetic field plotted in Fig. 1). These numbers are consistent with typical mirror mode scales published in previous studies (Soucek et al., 2008; Horbury and Lucek, 2009).

\section{Ion distribution function processing}

The ion distributions are provided by the Hot Ion Analyzer (HIA) part of the CIS instrument in the form of particle counts in $22.5^{\circ} \times 22.5^{\circ}$ solid angle bins for 31 logarithmically spaced energy levels between $6 \mathrm{eV}$ and $29 \mathrm{keV}$ collected over one spin period of four seconds. The HIA instrument does not distinguish between ion species; we therefore consider all the registered particles as protons neglecting the possible contribution of $\mathrm{He}++$ ions. In this study we used the calibrated 3-D distributions in the form of phase space density (PSD) obtained from the Cluster Active Archive.

When studying the interaction between particles and electro-magnetic field, one is usually interested in the distribution function in a reference frame which is not moving with respect to the plasma. This represents a fundamental problem for the analysis of spacecraft data measured in the spacecraft frame of reference when the bulk plasma flow velocity is comparable or larger than the thermal velocities of the particles of interest. In our case, the plasma flow ve- locity is $v_{\mathrm{f}} \approx 120 \mathrm{~km} \mathrm{~s}^{-1}$ and the thermal velocity of magnetosheath protons corresponding to temperature $T_{\perp} \sim 2 \mathrm{MK}$ is $v_{\mathrm{t}} \approx 130 \mathrm{~km} \mathrm{~s}^{-1}$. As a result, the distribution observed in spacecraft frame is skewed and some of the thermal ions are shifted to the low energy range where low particle fluxes to the sensor result in poor measurements (Paschmann and Daly, 1998).

To overcome this issue we re-binned the 3-D distributions to 2-D pitch angle distributions, converting to plasma reference frame in the process. In the original 3-D CIS data, a range of energies and a central direction vector of incoming particles is assigned to each distribution bin. For each bin we first correct the bin energy for the effect of spacecraft potential by adding the energy lost by the incoming ions due to the electrostatic field of the spacecraft (the mean spacecraft potential measured by the EFW instrument (Gustafsson et al., 1997) for this event was $3.4 \mathrm{~V}$ ). The energy range corresponding to the bin is next converted to particle velocities and these are transformed to the plasma frame by subtracting the bulk plasma flow velocity vector, estimated for each distribution individually from the corresponding moment. This transformed distribution is then rebinned to a two-dimensional grid in pitch angle and energy coordinates, assuming cylindrical symmetry of the distribution around an axis parallel with magnetic field direction (gyrotropy). Bins with one or fewer particles were removed from the integration. In this way we obtained two-dimensional distributions with 16 angular bins $\left(11.25^{\circ}\right.$ wide) and 10 logarithmically spaced energy bins from $10 \mathrm{eV}$ to $1 \mathrm{keV}$. A similar procedure was used previously by for example Fazakerley et al. (1995).

Figure 2 shows the 2-D distribution averaged over the entire studied interval in the spacecraft frame (top) and in the plasma rest frame with bulk velocity subtracted in the re-binning process (bottom). Clearly, the above procedure yields a distribution function much more symmetric in the B-parallel and anti-parallel directions. The spacecraft frame pitch-angle distribution in the bottom panel is plotted to demonstrate the magnitude and character of this instrumental effect and to show that the coordinate transform is indeed required to obtain meaningful results.

The 2-D (or pitch-angle) distributions throughout this article are expressed as cuts of the 3-D distribution in the $v_{\|} \times v_{\perp}$ plane calculated from the full 3-D distribution by assuming gyrotropy and averaging over the azimuthal angle. The energy range from $10 \mathrm{eV}$ to $1 \mathrm{keV}$ was chosen because outside this range, HIA particle counts were too low to allow reliable PSD estimates (on the high energy end due to lack of particles, on the low energy end due to low particle fluxes).

\section{Properties of the ion distribution}

To identify and describe the fine features of ion distribution, such as the trapped particles, one needs a reference "background" distribution function to compare against. While 


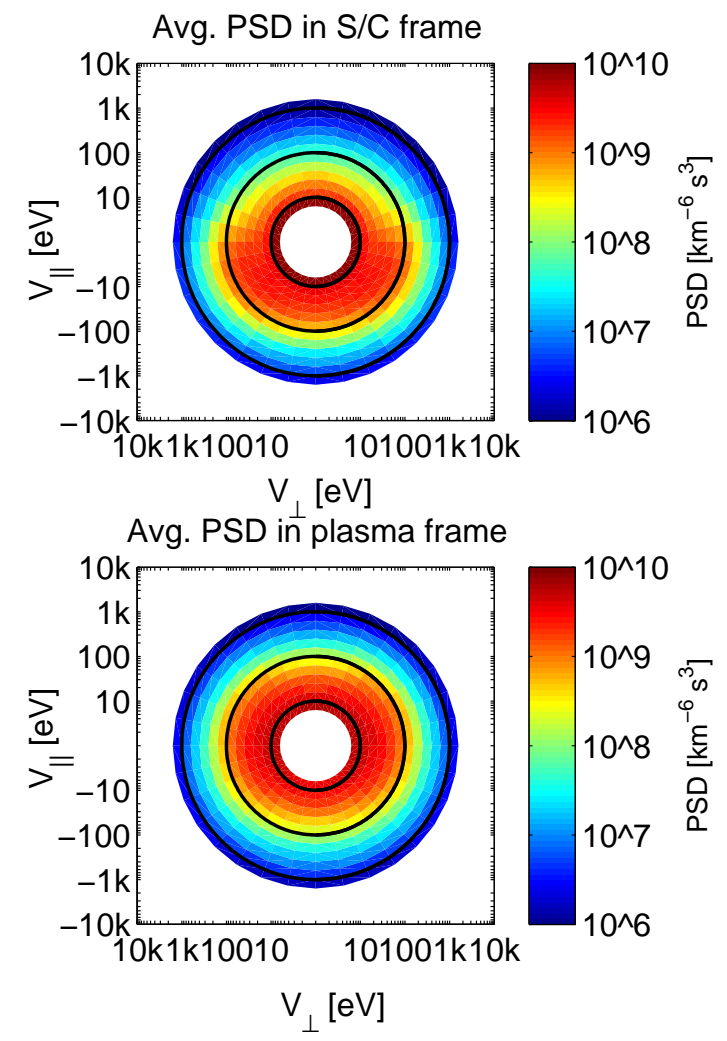

Fig. 2. Average re-binned two-dimensional cut of 3-D distributions for the entire interval. Top: original distribution in spacecraft reference frame. Bottom: the same distribution after transformation to plasma rest frame. Distributions are symmetric around vertical axis (gyrotropy was assumed in the re-binning process).

many theoretical and numerical studies of mirror modes use a two temperature bi-maxwellian distribution as the basic model for magnetosheath plasmas, direct spacecraft measurements have shown that this model is rarely applicable to real world magnetosheath plasmas. Since early spacecraft observations a suprathermal ion "tail" has been identified in magnetosheath plasmas (Formisano et al., 1973; Sanders et al., 1981). The integrated one-dimensional ion distribution functions presented in these and later studies exhibit an approximately power law shape above energies of few hundred eV. Formisano et al. (1973) modelled the distribution function by kappa distributions, while later studies used fits by a superposition of two Maxwellian populations (Sanders et al., 1981; Gosling et al., 1989; Tan et al., 1998). In particular, Gosling et al. (1989) divide the magnetosheath ions into a cooler core population of transmitted solar wind ions and a suprathermal shell with 4-10 times larger temperature and a fractional density of up to $20 \%$, composed of ions accelerated at the shock.

We followed this methodology and fitted the pitch-angle distributions in the plasma frame by a superposition of two bi-maxwellian distributions (a total of 6 free parameters
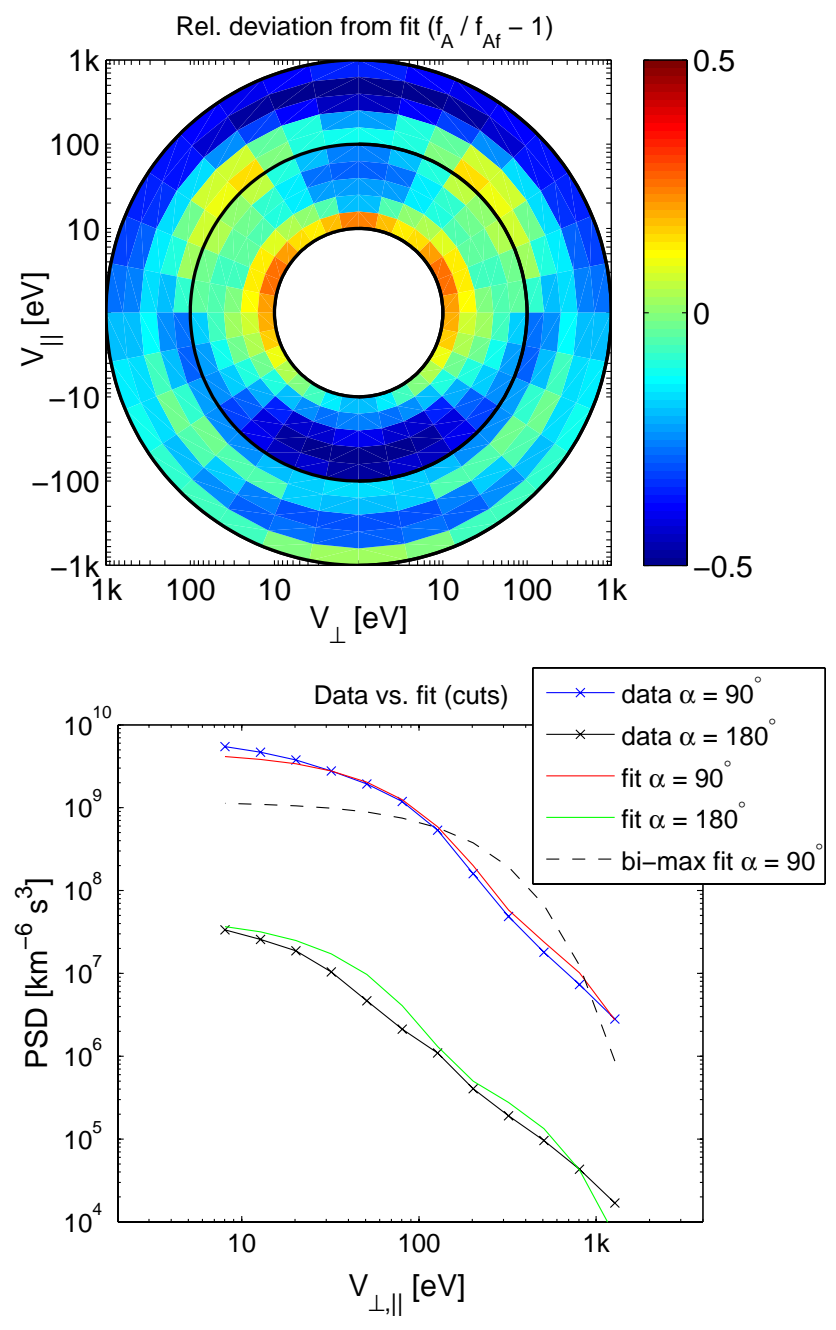

Fig. 3. Fits of the average distribution $f_{\mathrm{A}}$ by a double bimaxwellian model. Top: relative error of the best fit $\left(f_{\mathrm{A}}-f_{\mathrm{Af}}\right) / f_{\mathrm{Af}}$ for all pitch angle-energy bins (linear color scale). Bottom: cuts of the average 2-D distribution $f_{\mathrm{A}}$ and its double bi-maxwellian fit $f_{\text {Af }}$ for pitch angles $\alpha$ close to $90^{\circ}$ and $180^{\circ}$. For clarity, the parallel cuts are rescaled by a factor of 100 . Dashed line shows the best bi-maxwellian fit (perpendicular cut).

needed to be determined). The fits were performed using the standard Levenberg-Marquardt method (Press et al., 2007) with an initial approximation given by two bi-maxwellians independently fitted to distributions with reduced energy ranges: one with low energy bins $\left(E \leq E_{0}\right)$ and one with high energy bins $\left(E>E_{0}\right)$ only. To eliminate the effect of an adhoc choice of the initial energy ranges, the energy limit $E_{0}$ was varied between $50 \mathrm{eV}$ and $300 \mathrm{eV}$ and the best fit was used.

The above fitting procedure was applied to a mean ion distribution averaged over the entire 3 min interval $f_{\mathrm{A}}\left(v_{\|}, v_{\perp}\right)$ to obtain a fitted background distribution $f_{\text {Af. }}$. The results are shown in Fig. 3. The top panel shows the relative error of the best fit $\left(f_{\mathrm{A}}-f_{\mathrm{Af}}\right) / f_{\mathrm{Af}}$ and the bottom panel the comparison 
FGM B-field C3, tstart $=2004-03-18$ 07:23:30.000

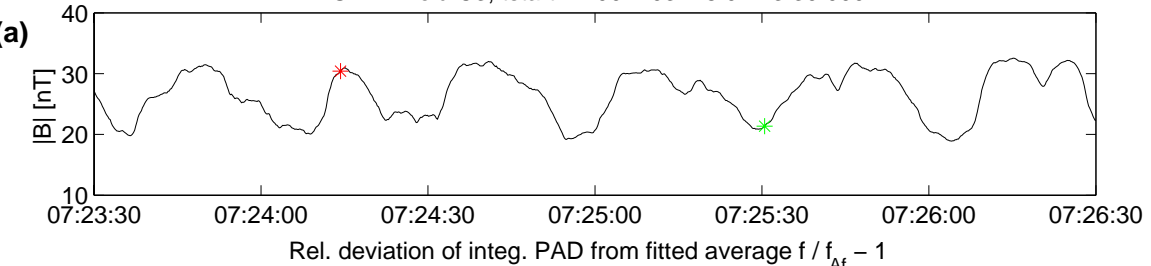

(b)

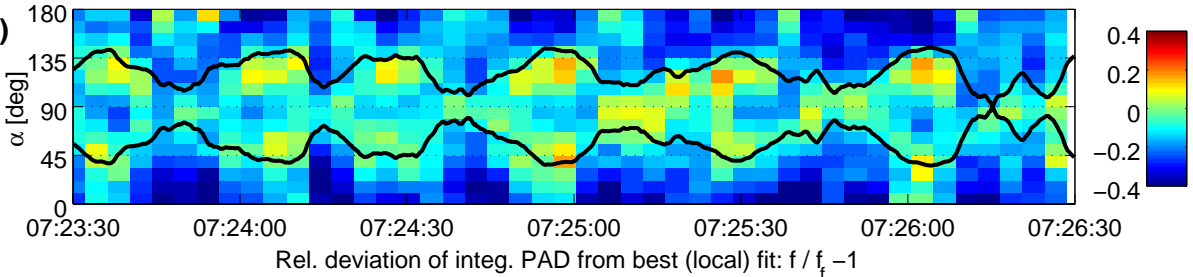

(c)

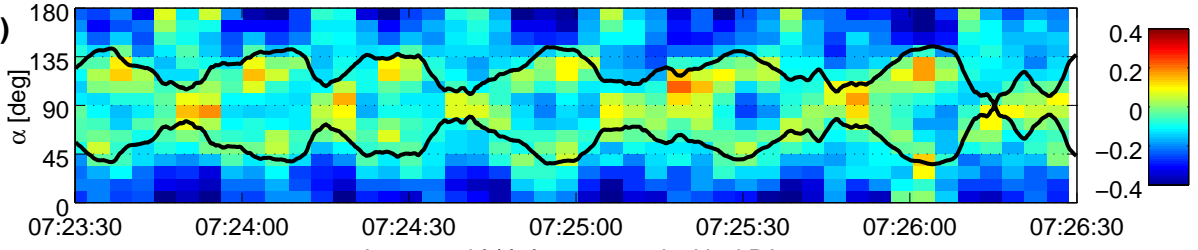

(d)

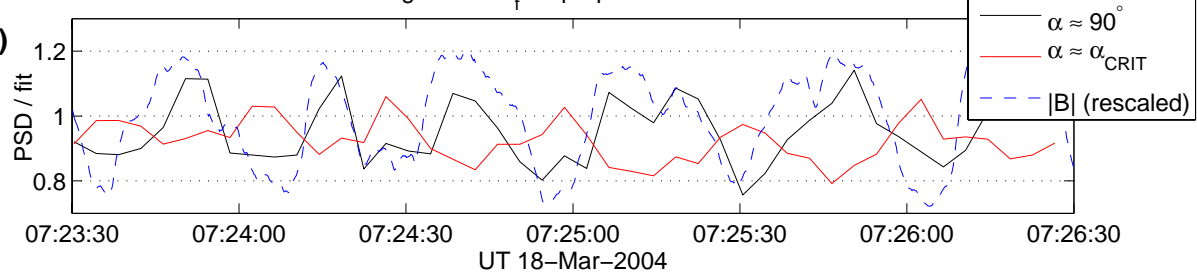

Fig. 4. Pitch angle distribution spectrograms integrated in energy $(10 \mathrm{eV}-1 \mathrm{keV})$. Top to bottom: (a) magnetic field magnitude. (b) Relative deviation of measured 2-D distribution from the fitted background $f_{\text {Af }}$ (linear color). Overplotted black line is the critical angle $\alpha_{\mathrm{c}}$. (c) Relative deviation of measured 2-D distribution from the local fit $f_{\mathrm{f}}$ (linear color). (d) The pitch angle distribution normalized by local fit $f / f_{\mathrm{f}}$ integrated over near-perpendicular pitch angles (black line) and pitch angles near $\alpha_{\mathrm{c}}$ (red line). Rescaled magnetic field magnitude $|B|$ is overplotted (blue dashed line).

of the mean distribution with the fit for cuts in perpendicular and parallel directions. The error of the fit is typically under $30 \%$ over the entire energy and pitch-angle range. For comparison, fits by a simple bi-maxwellian and kappa distributions were tried as well. The best bi-maxwellian fit in the least square sense is shown in Fig. 3 (dashed line), demonstrating the inapplicability of a bi-maxwellian model to this case. Consistently with e.g. Formisano et al. (1973), the fit by kappa distribution (not shown) gives a relatively good agreement, but the double bi-maxwellian model was found to be superior.

The double bi-maxwellian fit to the mean distribution yields a cold population with temperatures $T_{c \perp}=0.67 \mathrm{MK}$ $(58 \mathrm{eV})$ and $T_{\mathrm{c} \|}=0.35 \mathrm{MK}(30 \mathrm{eV})$. The suprathermal "shell" population represents about $4 \%$ of the total ion density and its estimated temperatures are $T_{\mathrm{h} \perp}=4.2 \mathrm{MK}$ $(360 \mathrm{eV})$ and $T_{\mathrm{h} \|}=3.0 \mathrm{MK}(260 \mathrm{eV})$. These results are close to the values obtained by Gosling et al. (1989).

\subsection{Trapped particles associated with field oscillations}

We can now analyze the variation of the ion distribution by investigating its deviation from the background fitted distribution $f_{\text {Af }}$. While $f_{\text {Af }}$ is not a true background distribution in the sense of describing unperturbed plasma, it serves well the purpose of providing a consistent and physically acceptable background against which local features (in particular the trapped particles) will stand out.

Figure 4 shows the main result of our analysis. The panel (b) shows the relative deviation of each measured pitch-angle distribution function from the fitted background $\left(f-f_{\text {Af }}\right) / f_{\text {Af }}$ integrated over the entire energy range $(10 \mathrm{eV}$ to $1 \mathrm{keV})$. A clear pattern is apparent in the plot: inside the troughs (close to B-field minima), we observe an increased PSD at intermediate pitch angles $40^{\circ}$ to $70^{\circ}$ and symmetrically from $110^{\circ}$ to $150^{\circ}$, the ion population at the most perpendicular pitch angles close to $90^{\circ}$ is, on the other hand, depleted. Close to the maxima of magnetic field, we observe the opposite effect: there is a mild enhancement in ion PSD 

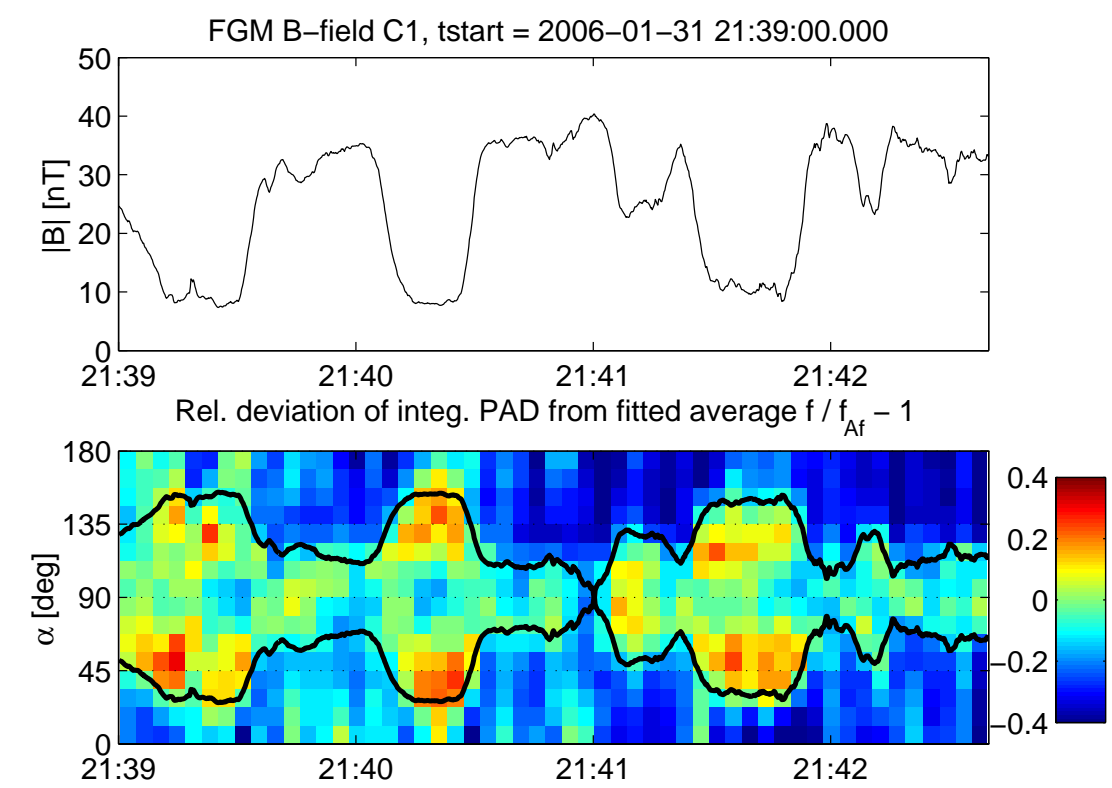

Fig. 5. Pitch angle distribution spectrogram integrated in energy for a second Cluster magnetosheath mirror mode event (31 January 2006). The panels correspond to panels (a) and (b) of Fig. 4.
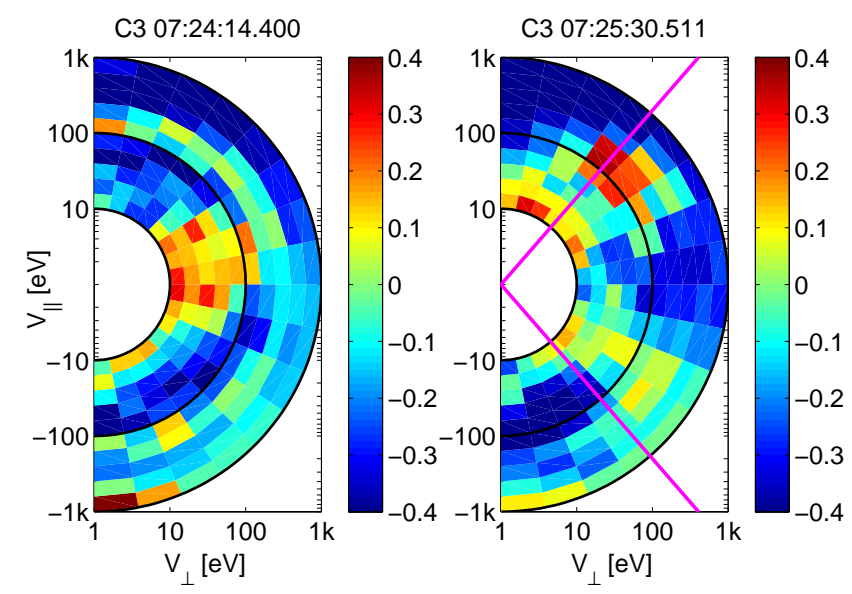

Fig. 6. Examples of the full 2-D distributions measured close to field maximum (left) and minimum (right). The distributions are plotted as relative deviation from the fitted background ( $f-$ $\left.f_{\text {Af }}\right) / f_{\text {Af }}$. The locations where the two measurements were taken correspond to asterisks in the top panel of Fig. 4. The magenta lines in the second plot indicate the calculated critical pitch angle $\alpha_{c}$.

at the perpendicular pitch angles $\left(\alpha \approx 90^{\circ}\right)$ and a depletion at the intermediate pitch angles.

Panel (c) shows a similar spectrogram, but in this case we plot the deviation of the measured distribution from the best local double bi-maxwellian fit $f_{\mathrm{f}}$. The difference from the previous panel is that in this case each distribution collected over one spacecraft spin $f$ is fitted with a double bimaxwellian model and this fit $f_{\mathrm{f}}$ is used as the reference. This plot therefore quantifies how well each distribution can be fitted by a double bi-maxwellian and which pitch angles contribute most to the discrepancy. In this plot, the same pattern can be seen, only the increase in PSD at perpendicular pitch angles, associated with field maxima, is more pronounced. Looking only at panel (b), one could suspect that this perpendicular particle population enhancement simply corresponds to an enhancement in the colder core ion population density inside the troughs. This panel shows that this population is indeed a non-thermal feature that cannot be properly modelled by the double bi-maxwellian distribution.

The above pattern resembles closely the signature of the trapped ion populations predicted from kinetic theory by Southwood and Kivelson (1993) for the case of linear mirror instability and in a follow-up paper (Kivelson and Southwood, 1996) for nonlinear large amplitude mirror structures. Kivelson and Southwood (1996) propose that particles with pitch angles $\alpha$ greater than a critical angle $\alpha_{\mathrm{c}}$ (in the sense of being more perpendicular or closer to $90^{\circ}$, defined as $\sin ^{2} \alpha>\sin ^{2} \alpha_{\mathrm{c}}=B / B_{\max }$ ) become trapped inside the magnetic troughs of the mirror structure and as the mirror wave grows, they gain energy by Fermi acceleration. It is further proposed that to saturate the growth and to attain marginal stability, the trapped population at perpendicular pitch angles must be non-adiabatically cooled. To make a direct comparison, we overplotted the critical pitch angle $\alpha_{\mathrm{c}}=\sin ^{-1}\left(B / B_{\max }\right)^{1 / 2}$ on our pitch angle spectrograms (black lines in Fig. 4). Since all magnetic field maxima are at almost the same level, for the peak field value we used a maximum value of $|B|$ over the interval under consideration: $B_{\max }=32.3 \mathrm{nT}$. It can be seen that the enhancements of the ion population inside the troughs are located precisely 
at the pitch angles slightly more perpendicular than $\alpha_{\mathrm{c}}$ (a direct comparison can be made to Fig. 7 of Kivelson and Southwood, 1996). More discussion is given in Sect. 4.

To demonstrate further the correlation with the magnetic field, in the bottom panel we integrated the ratio between the distribution $f$ and a local fit $f_{\mathrm{f}}$ over $\alpha$ between $75^{\circ}$ and $105^{\circ}$ and for $\alpha$ close to the critical angle (between $\alpha_{\mathrm{c}}$ and $\alpha_{\mathrm{c}}+20^{\circ}$ and analogously for the other pitch angle quadrant). The two integrated products are plotted in the bottom panel, clearly confirming the correlation between the magnetic field magnitude and the variation of the two trapped populations.

An analogous plot for a different mirror mode event observed by Cluster is shown in Fig. 5. Here only two panels, corresponding directly to panels (a) and (b) of Fig. 4, are plotted. This plot gives an additional example of the trapped ion signature, in this case even more pronounced, due to larger scale of the structures. Similar features were observed by Cluster for many other intervals, but the procedure described in this paper only produces clean results if the structures are sufficiently wide and the plasma flow velocity is relatively low. Only in such cases is the time resolution of the CIS instrument sufficient to perform several measurements of ion distribution within each peak or trough.

Figure 6 gives two examples of pitch angle distributions plotted again as deviations from the fitted background $f_{\mathrm{Af}}$, showing the details of the trapped particles close to magnetic field minima and maxima. The exact times when these measurements were taken are marked by asterisks in the top panel of Fig. 4. The features discussed above are clearly seen in these examples.

To provide the complete picture of the character of ion distribution function associated with mirror modes, in Fig. 7 we plot the same distributions in the form of the full phase space density (PSD). In addition to the examples corresponding to a peak and a trough, we also plot the PSD corresponding to the fitted average distribution $f_{\mathrm{Af}}$. In the right column are shown cuts of each distribution at several pitch angles relevant to the discussion. Comparison of the cuts at perpendicular and intermediate $\left(\alpha=120^{\circ}\right)$ pitch angles (green and black curves) reveals a strong enhancement of the distribution at perpendicular pitch angles near the peaks (panel a). However, the trapped particle population inside the troughs, clearly seen between the magenta lines in panel (b), is close to isotropic and the difference between the PSD of the heated particles (black) and the cooled particles at $\alpha \approx 90^{\circ}$ (green) is small. This possibly not self-evident feature has to be interpreted with reference to the background distribution shown in the panel (c). In the figure we observe that $f\left(|v|, \alpha=90^{\circ}\right)>$ $f\left(|v|, 120^{\circ}\right)$, as can be expected for a distribution well described by the double bi-maxwellian model and anisotropic in temperature $\left(T_{\perp}>T_{\|}\right)$. Comparing panels (b) and (c), one can verify that the population at intermediate pitch angles in panel (b) is significantly enhanced with respect to the background distribution, but the corresponding PSD does not necessarily exceed that of the cooled particles at $\alpha \approx 90^{\circ}$. Even
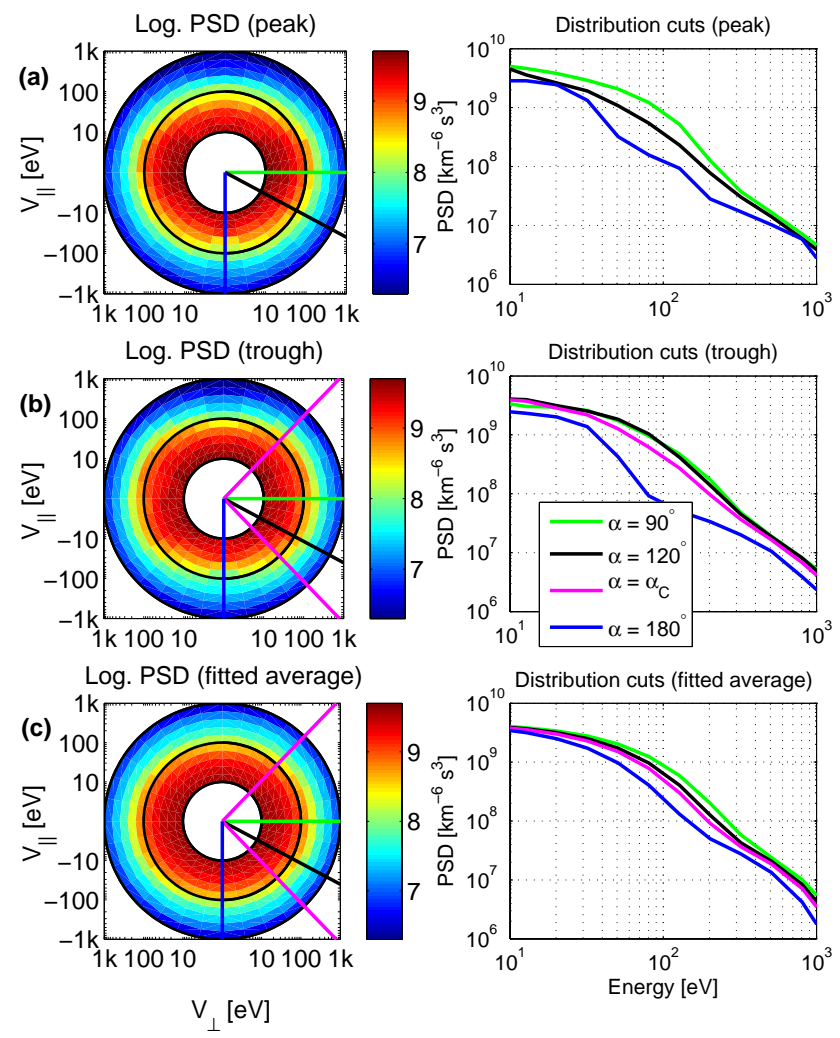

Fig. 7. Examples of the full 2-D distributions plotted as phase space density (without any normalization). The top panels show the ion distribution measured close to field maximum (a) and minimum (b), corresponding to those in Fig. 6. The bottom panels (c) show the fitted distribution $f_{\text {Af }}$. The right column shows cuts of the PSD at selected pitch angles; the respective pitch angles are visualized by lines of the same color in the corresponding 2-D distributions.

though the original unperturbed ion distribution is unknown and $f_{\text {Af }}$ only provides an approximation, this unperturbed distribution had to exhibit this kind of temperature anisotropy $T_{\perp}>T_{\|}$necessary for the mirror instability to develop.

\subsection{Energies of trapped particles}

One interesting and unexpected property seen in Fig. 6 is the distribution of trapped ion populations in energy. While the enhanced trapped population at intermediate angles inside the magnetic troughs spans over most of the considered energy range, the enhancement in particles at $\alpha \approx 90^{\circ}$ is only seen at lower energies (here below $100 \mathrm{eV}$ ).

Next we investigated whether this effect is systematic over the whole interval under study. In Fig. 8 we show the same normalized integrated pitch angle spectra as in panel (b) of Fig. 4, only this time the pitch angle distributions were integrated separately over low energies $(10 \mathrm{eV}-100 \mathrm{eV})$ and higher energies $(100 \mathrm{eV}-1 \mathrm{keV})$. The results confirm the initial impression from Fig. 6 . The trapped heated particles inside the troughs are clearly seen in both panels, while there 


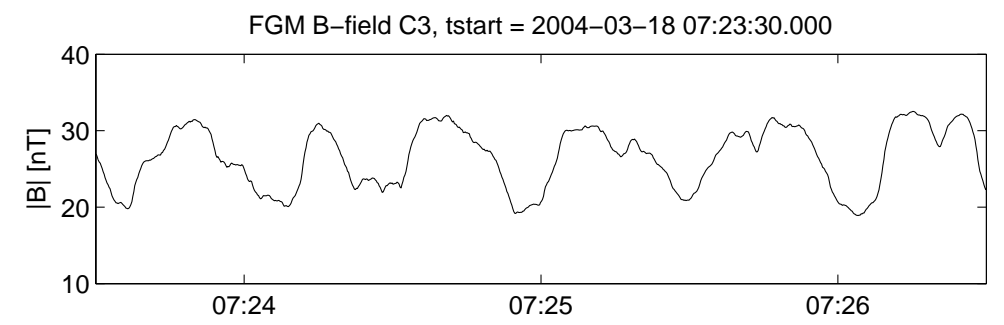

Rel. deviation of from fitted average $(10 \mathrm{eV}<\mathrm{E}<100 \mathrm{eV})$

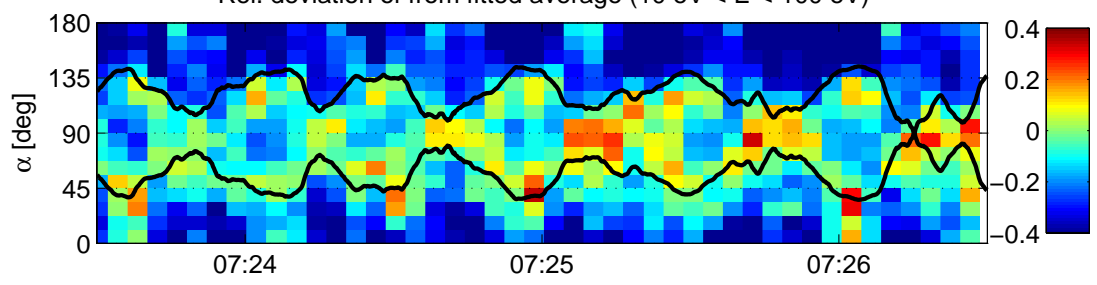

Rel. deviation of from fitted average $(100 \mathrm{eV}<\mathrm{E}<1 \mathrm{keV})$

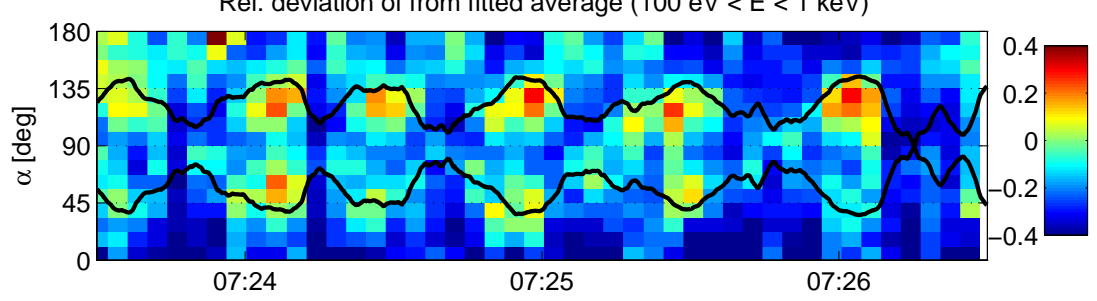

Fig. 8. Pitch angle distribution spectrograms integrated in low (middle panel) and high energy bands (bottom panel). Distributions are plotted as relative deviation of measured integrated 2-D distribution from the fitted background $f_{\mathrm{Af}}$ (linear color scale).

is no trace of the enhancement at $\alpha \approx 90^{\circ}$ corresponding to field maxima in the high energy plots.

\section{Discussion and conclusions}

The results presented in the previous section clearly demonstrate the presence of distinct populations of trapped ions inside the magnetic troughs and close to the field maxima. In the troughs we observed an enhancement at intermediate pitch angles around and above the critical pitch angle $\alpha_{\mathrm{c}}$ and at the magnetic field maxima we observe an enhancement in the perpendicular ion population $\alpha \approx 90^{\circ}$, primarily at energies below $100 \mathrm{eV}$.

Our results experimentally confirm the existence and properties of trapped ion populations predicted by theory and numerical simulations. When discussing mirror modes, it is important to make a distinction between small amplitude mirror waves, where the structure has a periodic character and where linear or quasi-linear theory is applicable, and between large amplitude non-linear structures (like those studied in this work), where linear approximations are in general violated and the field oscillations often have a form of coherent non-periodic structures.

The case of linear mirror modes has now been relatively well understood. The importance of trapped particles at small $v_{\|}$for the development of the instability has first been recognized by Southwood and Kivelson (1993) who predicted the depletion of ions at $\alpha \approx 90^{\circ}$ inside the magnetic troughs and the enhancement of this part of distribution near the peaks. The model is based on the different behavior of trapped and non-trapped particles and conservation of magnetic moment (betatron acceleration/deceleration). The model was later improved by Pantellini and Schwartz (1995) taking into account electric fields induced by warm electrons, but the essential features regarding trapped ions remained the same. This effect was later confirmed by quasi-linear theory and numerical simulations (Califano et al., 2008; Hellinger et al., 2009; Pokhotelov et al., 2010, and references therein). Although the predictions are based on approximations assuming small amplitude mirror structures and near-threshold instability, we observed both the enhancement and depletion of ions in association with nonlinear mirror structures.

The evolved non-linear stage of mirror instability is far more complex. Kivelson and Southwood (1996) developed a model of dynamic behavior of particles driven by growing large amplitude mirror structures. They predict that in this case, the ions trapped inside magnetic troughs at intermediate pitch angles can be heated by Fermi acceleration as the mirror structure grows and the mirror points of the particles move closer together. In the same manner, the deeply trapped particles at perpendicular pitch angles will be cooled, since their mirror points move apart during the development of the instability and/or due to magnetic moment conservation 
invoked in the linear case. For a more detailed discussion, see also Pantellini (1998). As shown in the previous section, we clearly observe both effects inside the troughs (Fig. 4). This effect was later observed in numerical simulations and derived (under restrictive assumptions) from quasi-linear theory (Califano et al., 2008).

One effect we observe and which is not addressed by nonlinear theory and simulations is the enhanced ion population at $\alpha \approx 90^{\circ}$ correlated with magnetic peaks, found primarily at low energies. Two explanations of this effect can be proposed. In the first scenario, the enhancement is explained by betatron acceleration, the same effect proposed by Southwood and Kivelson (1993) to create such an enhancement in small amplitude growing mirror waves. Here the particles with very small $v_{\|}$cannot move out of the high field region fast enough and experience the field enhancement associated with wave growth. Due to conservation of magnetic moment $\mu=m v_{\perp}^{2} / 2 B$, their $v_{\perp}$ is increased. This interpretation can also explain why the increase is seen only at low energies. At higher energies, the pitch angle range required to keep the particles within the high field region for a sufficiently long time becomes too narrow to be observed. A simple quantitative estimate of the maximum parallel velocity where we can observe this heating can be obtained as $v_{\|} \leq \gamma L_{\|}$, where $\gamma$ is the growth rate of the instability and $L_{\|}$ is the B-parallel scale of the structure. Assuming that a typical scale of the structure in the parallel direction is $1000 \mathrm{~km}$ (Sect. 2) and estimating the growth rate of the instability from observed plasma parameters as $\gamma \approx 0.02 \mathrm{~s}^{-1}$ (Gary et al., 1993), we obtain a condition $v_{\|} \leq 20 \mathrm{~km} \mathrm{~s}^{-1} \approx 2.1 \mathrm{eV}$. The range of pitch angles where betatron heating could occur is thus between $78^{\circ}$ and $102^{\circ}$ for a total ion energy of $50 \mathrm{eV}$, while at $500 \mathrm{eV}$ the range is much narrower $\left(86^{\circ}-94^{\circ}\right)$. These numbers are comparable with what is observed in Fig. 6, although the enhancement is observed at a wider range of pitch angles.

The second interpretation assumes that the particles observed near the peaks are the heated particles trapped inside the troughs, only they are observed very close to the mirror point, where their pitch angle is close to $90^{\circ}$. From the conservation of magnetic moment $\mu$ we can easily derive that the particles observed inside the trough very close to the critical angle $\alpha_{\mathrm{c}}$ are shallowly trapped and their mirror points lie very close to the field maximum. In Fig. 4 one observes that the enhancements inside the troughs are often found at $\alpha \approx \alpha_{\mathrm{c}}$ (within the resolution of the data). These particles hence propagate near the field maxima and the enhancements in perpendicular pitch angles around the peaks and near the critical angle in the troughs may represent signatures of the same particle population observed under different conditions. The two above interpretations are both consistent with the data and only differ in the mechanism responsible for the perpendicular heating of these particles, a property which is difficult to infer from our data and which depends primarily on the (unknown) characteristic rate of temporal change of the field intensity. Applying the Occam's razor principle, the latter interpretation appears more natural.

The existence and importance of these trapped ions has been discussed by numerous theoretical and numerical studies, including those discussed above, but to our knowledge only a single previously published study (Leckband et al., 1995) to some extent identified these features in spacecraft data. In this work the authors used ion data from the AMPTE-UKS satellite and calculated the difference between mean distributions associated with peaks and troughs averaged over $20 \mathrm{~min}$ of data where small amplitude "linear" mirror waves were observed. They noted that the difference is positive for small $v_{\|}$and negative in the rest of the phase space, consistently with predictions of Southwood and Kivelson (1993). The problem was independently approached by Fazakerley et al. (1995) by direct comparison of individual ion distributions inside mirror peaks and troughs, but the authors were unable to find any conclusive signatures of the trapped particles in the data. A clearer evidence of the modification of particle distribution by mirror structures has been published for the case of electrons (Chisham et al., 1998). While electrons are trapped and heated/cooled in a manner very similar to ions, their contribution to the dynamics of the instability is much weaker than that of ions.

Further experimental studies exist on the subject, where trapped particles are discussed, but the observed ion properties are only analyzed using moments of the distribution: density, perpendicular and parallel temperatures. Recent studies (Balikhin et al., 2009, 2010) demonstrate the increase in plasma temperatures associated with magnetic troughs and argue that adiabatic invariants are not conserved in this process and that trapped particle dynamics needs to be considered to explain the observations. Our study is consistent with this qualitative statement: the increase in both temperatures is also seen in our example and it is at least partly due to the enhancement in particle populations at intermediate pitchangles. Since this increase extends to much higher energies than the enhancement in $\alpha \approx 90^{\circ}$ particles at the magnetic peaks, it dominates the temperature variation in both perpendicular and parallel direction.

In our study we neglected the contribution of $\mathrm{He}++$ ions to the data and plasma dynamics. Magnetosheath plasma typically contains a small percentage $\left(n_{\alpha} / n_{\mathrm{p}}<5 \%\right)$ of Helium ions, but due to the measurement principle of Cluster CISHIA instrument we could not separate these from the proton population. In our dataset, they are erroneously counted as protons and may thus contribute to some of the noise and scatter in the figures. Due to their small relative concentration, this effect should not change the results significantly. The CODIF sensor of the CIS instrument could be in principle used to measure the separate proton and $\mathrm{He}++$ distributions, but such analysis is very complicated due to low detector counts and an overlap between proton and $\mathrm{He}++$ channels (Rème et al., 2001). It has been shown that even such a small fraction of Helium ions can modify the threshold and growth 
rate of mirror instability (Gary et al., 1993; Hellinger, 2007), but does not change the dynamics of the mirror instability qualitatively. The Helium ions could be trapped and heated in the same way as protons, but probably to a lesser extent due to their larger Larmor radius. We can thus safely assume that the results and conclusions of this study are not affected by the presence of $\mathrm{He}++$ population.

A final remark on the relevance of finite Larmor radius (FLR) effects should be made at this point. These effects, significant when the width of the structure is comparable to the ion Larmor radius, have been implicated in theoretical studies as being important for the non-linear saturation of mirror instability (Pokhotelov et al., 2010, 2005, and references therein). The gyro-radius of particles, depending on their energy and magnetic field level, is obviously largest deep inside the troughs. In our case it reaches maximum values of $r_{\mathrm{L}} \approx 70 \mathrm{~km}$ for $100 \mathrm{eV}$ ions and $r_{\mathrm{L}} \approx 200 \mathrm{~km}$ for $1 \mathrm{keV}$ ions. Since the narrowest trough in our dataset is about $600 \mathrm{~km}$ wide (according to the multi-spacecraft analysis mentioned in Sect. 2), the Larmor radius of trapped ions at the higher end of the energy spectrum is smaller, but comparable to the width of the troughs. This suggests that FLR effects can be important for the dynamics of fully evolved nonlinear mirror structures.

In our study we presented the first detailed measurements of the properties of trapped ions associated with non-linear magnetosheath mirror structures. Taking advantage of the superior resolution of Cluster data, we managed to identify their pitch angle variation dependent on the magnetic field and to show that both the predicted heating of ions at intermediate pitch angles and the cooling of trapped particles at small $v_{\|}$occurs inside the troughs of mirror structures.

Acknowledgements. We thank the Cluster principal investigators E. Lucek, I. Dandouras, M. André and their instrument teams for the data used in this study. We greatly appreciated the Cluster Active Archive facility, enabling an easy centralized access to full resolution calibrated Cluster data.

Guest Editor A. Masson thanks S. Schwartz and another anonymous referee for their help in evaluating this paper.

\section{References}

Balikhin, M. A., Sagdeev, R. Z., Walker, S. N., Pokhotelov, O. A., Sibeck, D. G., Beloff, N., and Dudnikova, G.: THEMIS observations of mirror structures: Magnetic holes and instability threshold, Geophys. Res. Lett., 36, L03105, doi:10.1029/2008GL036923, 2009.

Balikhin, M. A., Pokhotelov, O. A., Walker, S. N., Boynton, R. J., and Beloff, N.: Mirror mode peaks: THEMIS observations versus theories, Geophys. Res. Lett., 37, L05104, doi:10.1029/2009GL042090, 2010.

Balogh, A., Carr, C. M., Acuña, M. H., Dunlop, M. W., Beek, T. J., Brown, P., Fornacon, K.-H., Georgescu, E., Glassmeier, K.H., Harris, J., Musmann, G., Oddy, T., and Schwingenschuh, K.: The Cluster Magnetic Field Investigation: overview of in-flight performance and initial results, Ann. Geophys., 19, 1207-1217, doi:10.5194/angeo-19-1207-2001, 2001.

Burlaga, L. F., Ness, N. F., and Acũna, M. H.: Trains of magnetic holes and magnetic humps in the heliosheath, Geophys. Res. Lett., 33, L21106, doi:10.1029/2006GL027276, 2006.

Califano, F., Hellinger, P., Kuznetsov, E., Passot, T., Sulem, P. L., and Trávníček, P. M.: Nonlinear mirror mode dynamics: Simulations and modeling, J. Geophys. Res., 113, A08219, doi:10.1029/2007JA012898, 2008.

Chisham, G., Burgess, D., Schwartz, S. J., and Dunlop, M. W.: Observations of electron distributions in magnetosheath mirror mode waves, J. Geophys. Res., 103, 26765-26774, doi:10.1029/98JA02620, 1998.

Erdôs, G. and Balogh, A.: Statistical properties of mirror mode structures observed by Ulysses in the magnetosheath of Jupiter, J. Geophys. Res., 101, 1-12, doi:10.1029/95JA02207, 1996.

Escoubet, C. P., Fehringer, M., and Goldstein, M.: Introduction: The Cluster mission, Ann. Geophys., 19, 1197-1200, doi:10.5194/angeo-19-1197-2001, 2001.

Fazakerley, A. N., Coates, A. J., and Dunlop, M. W.: AMPTEUKS Observation of Ion Velocity Distributions Associated with Magnetosheath Waves, Adv. Space Res., 15, 349-352, 1995.

Formisano, V., Moreno, G., Palmiotto, F., and Hedgecock, P. C.: Solar wind interaction with the earth's magnetic field. 1. Magnetosheath., J. Geophys. Res., 78, 3714-3730, doi:10.1029/JA078i019p03714, 1973.

Fuselier, S. A., Anderson, B. J., Gary, S. P., and Denton, R. E.: Inverse correlations between the ion temperature anisotropy and plasma beta in the Earth's quasi-parallel magnetosheath, J. Geophys. Res., 99, 14931-14936, doi:10.1029/94JA00865, 1994.

Gary, S. P., Fuselier, S. A., and Anderson, B. J.: Ion anisotropy instabilities in the magnetosheath, J. Geophys. Res., 98, 14811488, 1993.

Génot, V., Budnik, E., Hellinger, P., Passot, T., Belmont, G., Trávnícček, P. M., Sulem, P.-L., Lucek, E., and Dandouras, I.: Mirror structures above and below the linear instability threshold: Cluster observations, fluid model and hybrid simulations, Ann. Geophys., 27, 601-615, doi:10.5194/angeo-27-601-2009, 2009.

Gosling, J. T., Thomsen, M. F., Bame, S. J., and Russell, C. T.: Ion reflection and downstream thermalization at the quasi-parallel bow shock, J. Geophys. Res., 94, 10027-10037, doi:10.1029/JA094iA08p10027, 1989.

Gustafsson, G., Boström, R., Holback, B., Holmgren, G., Lundgren, A., Stasiewicz, K., Ahlen, L., Mozer, F. S., Pankow, D., Harvey, P., Berg, P., Ulrich, R., Pedersen, A., Schmidt, R., Butler, A., Fransen, A. W. C., Klinge, D., Thomsen, M., Falthammar, C.G., Lindqvist, P.-A., Christenson, S., Holtet, J., Lybekk, B., Sten, T. A., Tanskanen, P., Lappalainen, K., and Wygant, J.: The electric field and wave experiment for the Cluster mission, Space Sci. Rev., 79, 137-156, doi:10.1023/A:1004975108657, 1997.

Hasegawa, A.: Drift mirror instability in the magnetosphere, Phys. Fluids, 12, 2642-2650, 1969.

Hellinger, P.: Comment on the linear mirror instability near the threshold, Phys. Plasmas, 14, 082105, doi:10.1063/1.2768318, 2007.

Hellinger, P., Trávníček, P., Mangeney, A., and Grappin, R.: Hybrid simulations of the magnetosheath compression: Marginal stability path, Geophys. Res. Lett., 30, 1959, 
doi:10.1029/2003GL017855, 2003.

Hellinger, P., Kuznetsov, E. A., Passot, T., Sulem, P. L., and Trávníček, P. M.: Mirror instability: From quasi-linear diffusion to coherent structures, Geophys. Res. Lett., 36, L06103, doi:10.1029/2008GL036805, 2009.

Horbury, T. S. and Lucek, E. A.: Size, shape, and orientation of magnetosheath mirror mode structures, J. Geophys. Res., 114, A05217, doi:10.1029/2009JA014068, 2009.

Horbury, T. S., Lucek, E. A., Balogh, A., Dandouras, I., and Rème, H.: Motion and orientation of magnetic field dips and peaks in the terrestrial magnetosheath, J. Geophys. Res., 109, A09209, doi:10.1029/2003JA010237, 2004.

Kaufmann, R. L., Horng, J.-T., and Wolfe, A.: Large-amplitude hydromagnetic waves in the inner magnetosheath., J. Geophys. Res., 75, 4666-4676, 1970.

Kivelson, M. G. and Southwood, D. J.: Mirror instability II: The mechanism of nonlinear saturation, J. Geophys. Res., 101, 17365-17372, doi:10.1029/96JA01407, 1996.

Leckband, J. A., Burgess, D., Pantellini, F. G. E., and Schwartz, S. J.: Ion distributions associated with mirror waves in the Earth's magnetosheath, Adv. Space Res., 15, 345-348, 1995.

McKean, M. E., Gary, S. P., and Winske, D.: Kinetic physics of the mirror instability, J. Geophys. Res., 98, 21313-21321, doi:10.1029/93JA01993, 1993.

Pantellini, F. G. E.: A model of the formation of stable nonpropagating magnetic structures in the solar wind based on the nonlinear mirror instability, J. Geophys. Res., 103, 4789-4798, doi:10.1029/97JA02384, 1998.

Pantellini, F. G. E. and Schwartz, S. J.: Electron temperature effects in the linear proton mirror instability, J. Geophys. Res., 100, 3539-3549, 1995.

Paschmann, G. and Daly, P. W. (Eds.): Analysis Methods for MultiSpacecraft Data, ISSI/ESA, Amsterdam, 1998.

Pokhotelov, O. A., Balikhin, M. A., Sagdeev, R. Z., and Treumann, R. A.: Halo and mirror instabilities in the presence of finite Larmor radius effects, J. Geophys. Res., 110, A10206, doi:10.1029/2004JA010933, 2005.

Pokhotelov, O. A., Sagdeev, R. Z., Balikhin, M. A., Onishchenko, O. G., and Fedun, V. N.: Nonlinear mirror waves in nonMaxwellian space plasmas, J. Geophys. Res., 113, A04225, doi:10.1029/2007JA012642, 2008.

Pokhotelov, O. A., Sagdeev, R. Z., Balikhin, M. A., Fedun, V. N., and Dudnikova, G. I.: Nonlinear Mirror and Weibel modes: peculiarities of quasi-linear dynamics, Ann. Geophys., 28, 21612167, doi:10.5194/angeo-28-2161-2010, 2010.

Press, W. H., Teukolsky, S. A., Vettering, W. T., and Flannery, B. P.: Numerical Recipes: The Art of Scientific Computing, 3rd edition, Cambridge University Press, New York, 2007.

Rème, H., Bosqued, J. M., Sauvaud, J. A., Cros, A., Dandouras, J., Aoustin, C., Bouyssou, J., Camus, T., Cuvilo, J., Martz, C., Medale, J. L., Perrier, H., Romefort, D., Rouzaud, J., D‘Uston, C., Mobius, E., Crocker, K., Granoff, M., Kistler, L. M., Popecki, M., Hovestadt, D., Klecker, B., Paschmann, G., Scholer, M., Carlson, C. W., Curtis, D. W., Lin, R. P., McFadden, J. P., Formisano, V., Amata, E., Bavassano-Cattaneo, M. B., Baldetti, P., Belluci, G., Bruno, R., Chionchio, G., di Lellis, A., Shelley, E. G., Ghielmetti, A. G., Lennartsson, W., Korth, A., Rosenbauer, H., Lundin, R., Olsen, S., Parks, G. K., McCarthy, M., and Balsiger, H.: The Cluster Ion Spectrometry (CIS) Experiment,
Space Sci. Rev., 79, 303-350, doi:10.1023/A:1004929816409, 1997.

Rème, H., Aoustin, C., Bosqued, J. M., Dandouras, I., Lavraud, B., Sauvaud, J. A., Barthe, A., Bouyssou, J., Camus, Th., CoeurJoly, O., Cros, A., Cuvilo, J., Ducay, F., Garbarowitz, Y., Medale, J. L., Penou, E., Perrier, H., Romefort, D., Rouzaud, J., Vallat, C., Alcaydé, D., Jacquey, C., Mazelle, C., d'Uston, C., Möbius, E., Kistler, L. M., Crocker, K., Granoff, M., Mouikis, C., Popecki, M., Vosbury, M., Klecker, B., Hovestadt, D., Kucharek, H., Kuenneth, E., Paschmann, G., Scholer, M., Sckopke, N., Seidenschwang, E., Carlson, C. W., Curtis, D. W., Ingraham, C., Lin, R. P., McFadden, J. P., Parks, G. K., Phan, T., Formisano, V., Amata, E., Bavassano-Cattaneo, M. B., Baldetti, P., Bruno, R., Chionchio, G., Di Lellis, A., Marcucci, M. F., Pallocchia, G., Korth, A., Daly, P. W., Graeve, B., Rosenbauer, H., Vasyliunas, V., McCarthy, M., Wilber, M., Eliasson, L., Lundin, R., Olsen, S., Shelley, E. G., Fuselier, S., Ghielmetti, A. G., Lennartsson, W., Escoubet, C. P., Balsiger, H., Friedel, R., Cao, J.-B., Kovrazhkin, R. A., Papamastorakis, I., Pellat, R., Scudder, J., and Sonnerup, B.: First multispacecraft ion measurements in and near the Earth's magnetosphere with the identical Cluster ion spectrometry (CIS) experiment, Ann. Geophys., 19, 1303-1354, doi:10.5194/angeo19-1303-2001, 2001

Russell, C. T., Riedler, W., Schwingenschuh, K., and Yeroshenko, Y.: Mirror instability in the magnetosphere of Comet Halley, Geophys. Res. Lett., 14, 644-647, doi:10.1029/GL014i006p00644, 1987.

Sanders, G. D., Freeman, J. W., and Maher, L. J.: A two-temperature plasma distribution in the magnetosheath at lunar distances, J. Geophys. Res., 86, 2475-2479, doi:10.1029/JA086iA04p02475, 1981.

Schwartz, S. J., Burgess, D., and Moses, J. J.: Low-frequency waves in the Earth's magnetosheath: present status, Ann. Geophys., 14, 1134-1150, doi:10.1007/s00585-996-1134-z, 1996.

Soucek, J., Lucek, E., and Dandouras, I.: Properties of magnetosheath mirror modes observed by Cluster and their response to changes in plasma parameters, J. Geophys. Res., 113, A04203, doi:10.1029/2007JA012649, 2008

Southwood, D. J. and Kivelson, M. G.: Mirror instability. I - Physical mechanism of linear instability, J. Geophys. Res., 98, 91819187, 1993.

Tajiri, M.: Propagation of Hydromagnetic Waves in Collisionless Plasma. II. Kinetic Approach, J. Physical Society of Japan, 22, 1482, 1967.

Tan, L. C., Fung, S. F., Kessel, R. L., Chen, S., Green, J. L., and Eastman, T. E.: Ion temperature anisotropies in the Earth's highlatitude magnetosheath: Hawkeye observations, Geophys. Res. Lett., 25, 587-590, doi:10.1029/98GL00306, 1998.

Tsurutani, B. T., Smith, E. J., Anderson, R. R., Ogilvie, K. W., Scudder, J. D., Baker, D. N., and Bame, S. J.: Lion roars and nonoscillatory drift mirror waves in the magnetosheath, J. Geophys. Res., 87, 6060-6072, 1982.

Tsurutani, B. T., Southwood, D. J., Smith, E. J., and Balogh, A.: Nonlinear magnetosonic waves and mirror mode structures in the March 1991 ULYSSES interplanetary event, Geophys. Res. Lett., 19, 1267-1270, doi:10.1029/92GL00782, 1992.

Tsurutani, B. T., Lakhina, G. S., Verkhoglyadova, O. P., Echer, E., Guarnieri, F. L., Narita, Y., and Constantinescu, D. O.: Magnetosheath and heliosheath mirror mode structures, interplanetary 
magnetic decreases, and linear magnetic decreases: Differences and distinguishing features, J. Geophys. Res., 116, A02103, doi:10.1029/2010JA015913, 2011.

Vedenov, A. A. and Sagdeev, R. Z.: Some properties of a plasma with an anisotropic ion velocity distribution in a magnetic field, in: Plasma Physics and the Problem of Controlled Thermonuclear Reactions, edited by: Leontovich, M. A., vol. 3, pp. 332339, Pergamon Press, New York, 1958.
Winterhalter, D., Neugebauer, M., Goldstein, B. E., Smith, E. J., Tsurutani, B. T., Bame, S. J., and Balogh, A.: Magnetic Holes in the Solar Wind and Their Relation to Mirror Mode Structures, Space Sci. Rev., 72, 201-204, doi:10.1007/BF00768780, 1995. 